\title{
Plasma dust as model crystals
}

\author{
Dust particles embedded in lonized plasma can be made to assemble into crystals of a kind, offering new routes with \\ possible astrophysical implications to the study of the crystalline state.
}

THAT physicists are the masters of artifice in the experiments they design is widely recognized, but sometimes their tricks quite take the breath away. Most of what follows is about an experiment of outstanding ingenuity which, despite the electronic equipment no doubt accompanying it, is also simple. The word 'elegant' is overworked, but in this case it applies.

First, a few words to explain why those concerned have bothered. The crystalline state has been amply studied in the past century or so, leading (among other things) to the modern electronics industry. But nature has been niggardly with the exemplars of the crystalline state it offers. In most crystals, atoms are separated by no more than a fraction of a nanometre. In ionic crystals such as sodium chloride, interatomic distances are no different from those in molecules. In metals, they are even smaller.

To the extent that the materials found useful in the real world are similar, it may be held that it does not matter very much that most studies of the crystalline state have been carried out with crystals in which the atoms are tightly packed. But that overlooks the need to test certain general properties of the crystalline state over a much wider range of parameters than in natural or even synthetic crystals.

How generally valid, for example, is the rule that the crystalline state becomes a liquid in a phase transition marked by the accumulation of disorder in an otherwise regularlattice? Or are the dislocations (which are, in a sense, ordered patterns of disorder) that reduce the strength of real solids below their theoretical strength also found in other kinds of crystals?

Such questions explain the eagerness with which solid-state physicists pounce on every newly discovered system in which there is an ordered array of entities in case it will be a good model of a real crystal. There are now several macroscopic systems that will serve. One is the now-familiar raft of soap bubbles on a liquid surface; that, at least, can simulate dislocations visually. More generally, Langmuir-Blodgett films consisting of unimolecular layers of some material on an immiscible liquid will simulate the melting transition, as will simple molecules adsorbed on a clean metal surface, but these are two-dimensional systems in which the melting transition is not a firstorder transition (meaning that there is no latent heat).

NATURE · VOL 370 • 11 AUGUST 1994
The same is true of the 'electron solids' found in thin layers of semiconductor material, where the transition from a solid to a gas is accompanied by a marked change of electrical properties but little else. Other models of the crystalline state include liquid crystals in which weakly interacting molecules are ordered relatively to each other both in space and orientation, and whose utility (in liquid displays) rests on the ease with which the state of order can be changed by a weak electrical field, and colloid solutions, where interparticle forces are usually too weak to make them good models of a crystal.

So why not set out to build another model? That is what a group of six people, five from Germany and one from the University of Iowa in the United States, have now done (Thomas, H. et al. Phys. Rev. Lett. $73,652 ; 1994)$. They call their crystal a 'plasma crystal' formed by the mutual electrostatic repulsion of particles of dust held within an ionized plasma. The authors note the obvious relevance of their study to what may happen in clouds of interstellar dust, which are also 'dusty plasmas', as well as to the practical problems of the contamination of newly formed surfaces by dust in microelectronics fabrication.

How to simulate such a crystal in the laboratory? The plasma is made from argon gas enclosed in a small vacuum chamber and excited by a radio-frequency discharge. The chamber also contains two electrodes, a lower circular electrode $8 \mathrm{~cm}$ in diameter and an upper electrode in the form of an annulus - a $10-\mathrm{cm}$ circle in which a $3-\mathrm{cm}$ hole has been cut. Both electrodes are arranged horizontally and are $2 \mathrm{~cm}$ apart.

For dust, Thomas et al. have used $7 \mu \mathrm{m}$ plastic spheres, which are dusted into the plasma chamber through the hole in the upper electrode. In the conditions of the experiment, the plastic spheres form a circular cloud some $3 \mathrm{~cm}$ in diameter. The positions of the dust particles are made visible by means of a fan-shaped laser beam (made by putting a cylindrical lens in front of a laser), when the scattered light can be seen (even by the naked eye, it appears) through the central hole in the upper electrode and also recorded by means of a charge-coupled device for numerical analysis.

What happens? In one run of the experiment described in detail, the dust grains are arranged in 18 layers all parallel with the horizontal electrodes. The positions of the particles within each layer are determined simply by shifting the laser beam vertically. Even to the eye, there is a high degree of regularity in the arrangement of the particles in a plane; many points of light form nearly perfect squares, others form trapezia with the same dimensions, but others appear to be missing altogether. The authors estimate that the inter-particle spacing is $250 \mu \mathrm{m}$, or $0.25 \mathrm{~mm}$.

The explanation is straightforward. One clue is that in the experiment described, the lower electrode acquires a negative electrical bias of $14 \mathrm{~V}$, which is enough to levitate the dust particles. The authors explain that the electrical charge on a particular dust particle will be determined by the competitive attachment of electrons and ions to its surface, but, in this experiment, the electrons win, the dust particles are negatively charged and, although they are presumably surrounded by partially neutralizing sheaths of argon ions (as in the Debye-Hückel picture of ions in aqueous solution), they repel each other electrostatically

How good is the model? There are now well-known techniques for telling whetheran array of dots in a two-dimensional plane embodies a degree of order: join each pair of neighbour-points with a straight line, bisect that line and draw a line at right-angles through the bisection point until it meets others like itself. The result is that each point is embedded in what is called a Voronoi cell. The most telling proof that Thomas et al. have indeed made a 'dusty plasma crystal' is that their distributions of particles in horizontal planes are strikingly more regular than would be obtained with a random distribution of points in a plane.

What happens next? The obvious lack in this first account is that there is no investigation of the three-dimensional properties of the dusty crystal. And a first attempt at observing the melting transitions (by increasing the radio-frequency power) seems to have been swamped by the large motions imparted to all the dust particles, but that test will ultimately hang on a way of feeding energy directly into the vibrational modes of the dusty crystal.

What the authors themselves would like to do is to carry out their experiment in 'microgravity' conditions. On the face of things, it is a more deserving candidate for room in some future Space Shuttle flight than most of the others so far suggested.

John Maddox 